\title{
Influence of universal HBV vaccination on chronic HBV infection in Italy: Results of a cross-sectional multicenter study
}

\author{
Evangelista Sagnelli $^{1}$ (D) | Tommaso Stroffolini $^{2}$ | Caterina Sagnelli ${ }^{1}$ | \\ Filomena Morisco $^{3}$ | Nicola Coppola ${ }^{1}$ | Antonina Smedile ${ }^{4}$ | \\ Mariantonietta Pisaturo $^{5}$ | Guido Colloredo ${ }^{6}$ | Sergio Babudieri ${ }^{7}$ | Anna Licata ${ }^{8}$ | \\ Giuseppina Brancaccio ${ }^{9}$ | Angelo Andriulli ${ }^{10}$ | Piero L. Almasio ${ }^{8}$ | \\ Giovani B. Gaeta $^{9}$ | on behalf of the EPACRON study group, ${ }^{,+\neq}$

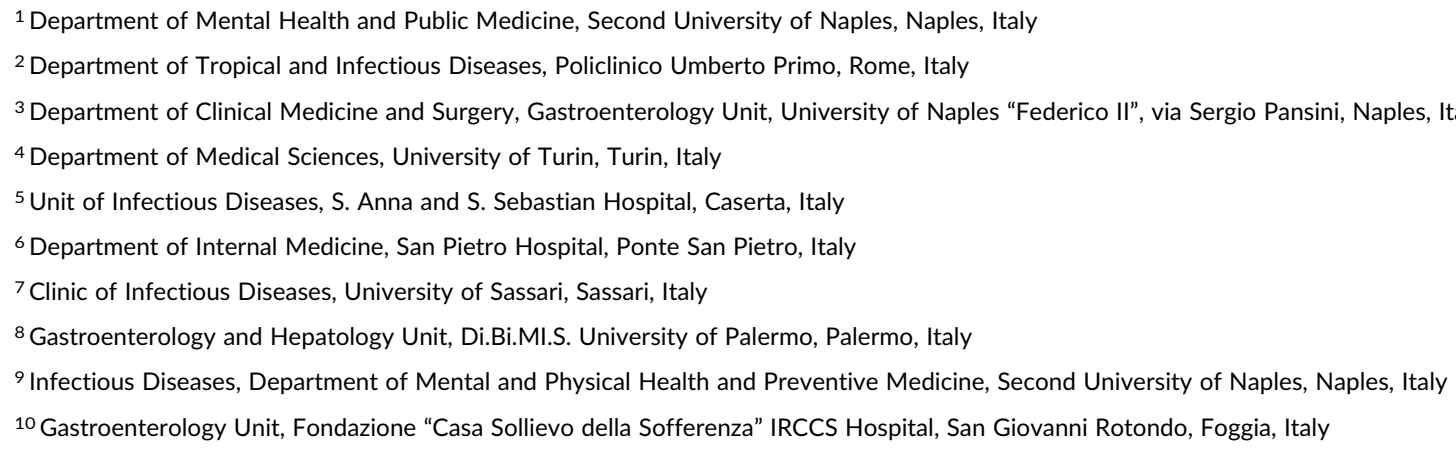

\section{Correspondence}

Evangelista Sagnelli. Department of Mental and Public Health, Section of Infectious Diseases, Second University of Naples, Largo Madonna delle Grazie n. 1, Naples, Italy.

Email: evangelista.sagnelli@unina2.it; evangelistasagnelli@libero.it

\section{Funding information}

Gilead Sciences, Grant number: An unrestricted grant for the study was provided $b$
Background and Aim: The universal hepatitis B vaccination for infants and 12-year-old adolescents (the latter limited to the first 12 years of application) was launched in Italy in 1991. Twenty-three years later we evaluated the impact of the vaccination campaign on the burden of HBsAg-positive chronic liver diseases (CLD).

Material and Methods: A total of $513 \mathrm{HBsAg-positive} \mathrm{chronic} \mathrm{carriers} \mathrm{referring} \mathrm{to} 16$ Italian liver units were investigated and compared with HBsAg carriers enrolled in previous surveys.

Results: The proportion of inactive carriers decreased from $20.0 \%$ in 2001 to $3.3 \%$ in 2014 , while that of cirrhotic patients increased from $22.6 \%$ to $33.2 \%$. Regarding the age class 0-33 (fully covered by HBV vaccination in 2014), the rate of inactive carriers decreased from the $21.7 \%$ in 2001 to $5.9 \%$ in 2014 , that of chronic hepatitis from

Abbreviations: CLD, chronic liver diseases; HCC, hepatocellular carcinoma; AFP,

a-fetoprotein; PCR, polymerase chain reaction.

${ }^{\dagger}$ Coordinating group: Piero Luigi Almasio, Giovanni Battista Gaeta, Evangelista Sagnelli, Tommaso Stroffolini.

${ }^{\ddagger}$ Peripheral centers: Angelo Andriulli, Sergio Babudieri, Giuseppina Brancaccio, Bruno Cacopardo, Guido Colloredo, Nicola Coppola, Massimo De Luca, Caterina Furlan, Anna Licata, Filomena Morisco, Mario Pirisi, Mariantonietta Pisaturo, Floriano Rosina, Maurizio Russello, Caterina Sagnelli, Teresa Santantonio, Antonina Smedile. 
$17.5 \%$ to $5.2 \%$ and that of cirrhosis cases from $26.4 \%$ to $4.1 \%$. Instead, in the over-60 age group the rate of inactive carriers increased from $22.8 \%$ to $41.2 \%$ and that of chronic hepatitis from $16.8 \%$ to $46 \%$; the rate of patients with cirrhosis ranged from $5 \%$ to $8 \%$ in different studies.

Conclusion: Twenty-three years after the introduction universal HBV vaccination in Italy, the clinical presentation of CLD had shown a shift toward older ages and more severe diseases.

\section{KEYWORDS}

chronic hepatitis B, HBsAg chronic carriers, HBsAg-positive chronic hepatitis, HBsAgpositive chronic hepatitis clinical presentation, $\mathrm{HBV}$ vaccination

\section{1 | INTRODUCTION}

Universal vaccination against HBV infection was introduced in Italy in the summer of 1991 for all infants and all 12-year-old adolescents (the latter limited to the first 12 years of the campaign). As a result, by 2014 virtually all Italians aged 0-33 years had been vaccinated against HBV. In the pre-vaccination era, two Italian multicenter retrospective surveys evaluated the etiology and clinical presentation of chronic liver diseases (CLD). The first found that $60 \%$ of 1154 patients enrolled from 1976 to 1981 were $\mathrm{HBsAg-positive}{ }^{1}$; the prevalence was $31.3 \%$ in a second survey performed on 5461 patients with CLD enrolled from 1980 to $1989 .^{2}$ In 2001, 10 years after the introduction of HBV universal vaccination, a prospective multicenter investigation on 9997 Italian patients with CLD showed that $23.2 \%$ were $\mathrm{HBsAg}$-positive. ${ }^{3}$ The above-mentioned studies indicate a substantial decrease in the impact of HBV etiology on CLD cases investigated in Italy from 1976 to 2001.

In 2014 a further survey on 2557 subjects with CLD was performed. ${ }^{4}$ The present paper describes the demographic and clinical data of the 513 subjects who were HBsAg-positive, and analyzes the changes in the demographics and clinical presentation occurring in Italy in the last 4 decades. More importantly, we analyzed the impact of universal HBV vaccination on the burden of HBsAg-positive CLD in Italy more than 23 years after its introduction.

\section{2 | PATIENTS AND METHODS}

The original database comprised all consecutive patients with CLD seen in 16 Italian liver units during the year 2014 as inpatients or outpatients. For the purpose of the present analysis, we retrieved all HBsAg-positive carriers aged over 18. Most of the participating liver units have cooperated in previous clinical investigations, and applied the same clinical approach and laboratory methods. The study design and method was described in detail in a previous study. 4

The diagnosis of chronic hepatitis was based on liver histology in nearly $80 \%$ of the cases and on the persistence of ALT elevation for
6 months or more in the remaining $20 \%$ in the absence of biochemical and ultrasound or transient evidence of liver cirrhosis. ${ }^{5}$ Liver cirrhosis was diagnosed based on liver histology or, in patients who did not undergo liver biopsy, on the presence of the peculiar clinical, biochemical, and ultrasound or transient signs. ${ }^{5}$ According to accepted criteria, hepatocellular carcinoma ( $\mathrm{HCC}$ ) was diagnosed based on histological and/or imaging findings and on serum a-fetoprotein (AFP) levels. ${ }^{6}$ The presence of serum HBsAg identified the HBV etiology, whereas the detection also of the antibody to HDV identified HBV/ HDV coinfection and the detection of the antibody to HCV identified $\mathrm{HBC} / \mathrm{HCV}$ coinfection; HBsAg positivity and alcohol abuse defined $\mathrm{HBV} /$ alcohol abuse. Alcohol abuse was defined as an intake of more than four alcoholic drinks per day over 5 years or more, whereas social drinking was a daily intake of 1-4 alcoholic beverages. ${ }^{7,8}$

An echo-assisted percutaneous liver biopsy was performed when required by the physician in care for diagnostic purposes. In each liver unit a skilled pathologist unaware of the clinical and laboratory data evaluated liver histology. In particular, liver necroinflammation and fibrosis were assessed by the Ishak ${ }^{9}$ or Metavir scoring system ${ }^{10}$ and standardized criteria were used to convert the Ishak scores to Metavir scores. ${ }^{11}$ Transient elastometry was performed by Fibroscan ${ }^{12-14}$ and used as an alternative method to assess liver fibrosis. ${ }^{12}$

Serum HBsAg and antibody to HCV, HDV, and HIV were sought using commercial immunoenzymatic assays. Plasma HBV DNA was determined by real-time polymerase chain reaction $(P C R)^{15}$ by this method, the detection limit in plasma samples is estimated at around $40 \mathrm{IU} / \mathrm{mL}$.

The collection of personal data was made in full compliance with the Italian law on personal data collection, and each patient gave his/her informed consent to participate in the study, for the collection and storage of biological material and for the use in clinical research of the data obtained. All procedures applied in the study were in accordance with current international guidelines, with the standards of human experimentation of the local Ethics Committees, and with the Helsinki Declaration of 1975, revised in 1983. At the time of first observation, each patient signed an informed consent for the collection of personal data, established in full agreement with the rules of the Ethics Committee of the 
coordinating center (A.O.U.P. of Palermo, Italy). Patients who agreed to undergo liver biopsy signed an appropriate informed consent before this procedure was performed. All patients were included only once, even if seen several times during the observation period. For each patient, demographic, etiological, and clinical data were recorded using a pre-coded questionnaire (Air-Tel Telematica, Italy). Due to the prevalent epidemiological nature of the study, data on the treatment of HBsAg-positive CLD were not collected. No patient refused to participate in the study.

\section{1 | Statistical analysis}

The data were collected in a pre-established electronic CRF database (web-based data collection, e-CRF provided by Air-Tel ${ }^{\circledR}$, Airon Telematica, Milan, ITALY). Categorical variables were summarized as absolute counts and proportions. Differences in proportion were evaluated by the chi square test or Fisher's exact test. A $P$-value $<0.05$ was considered to be significant. All $P$ values were two-tailed.

\section{3 | RESULTS}

The 513 patients with HBV chronic infection enrolled in the present study were prevalently males (sex ratio $=1.8$ ) and outpatients $(88.5 \%$ ), and $72.1 \%$ of cases came from southern Italy or one of the two major islands (Sicily or Sardinia). Eighty-four (16.4\%) were alcohol abusers, 42 (8.2\%) social drinkers, and 387 (75.4\%) abstainers. Only 17 cases (3.3\%) were HBV inactive carriers, 326 (63.4\%) had chronic hepatitis, 118 (23.0\%) had compensated cirrhosis, 28 (5.5\%) decompensated cirrhosis, and 24 (4.7\%) liver cirrhosis with superimposed $\mathrm{HCC}$ (Table1). The hepatitis $\mathrm{B}$ e antigen ( $\mathrm{HBeAg}$ ) was detected in 32 (6.2\%) patients, anti-HDV positivity in 61 (11.9\%), and anti-HCV positivity in 47 (10.7\%) out of the 440 tested (Table 2). HBV-DNA values were not analyzed, because of the possible influence of antiHBV treatments, which were not registered.

The original database consisted of 2557 patients with CLD, of whom the 513 (20.2\%) with HBV etiology are described in the present study. The database included also 1438 cases with HCV etiology, alone in 1286 (50.3\%) and with concomitant alcohol abuse in 152 (5.9\%). Alcohol abuse was the only etiologic agent of 163 cases (6.4\%), NAFLD/NASH of 162 (6.3\%), autoimmune hepatitis of 60 (2.3\%), and primary biliary cholangitis of $28(1.1 \%)$; hemochromatosis and Wilson's disease were rare diagnoses. In the remaining patients no etiologic agent was detected.

To identify changes occurring in the last 4 decades, we compared the data from the present study with those observed in HBsAg-positive patients investigated in 1976-81, 1980-89, 2001, and 2006-2007 (Table 3). The mean age of patients progressively increased from $27 \pm 16$ years in patients enrolled in the 70 s to $57.8 \pm 13.7$ in those observed in 2014 , but male preponderance remained relatively stable over time. The data on the percentages of $\mathrm{HBeAg}$, anti-HDV, and anti-HCV-positive cases were not available for patients enrolled in 1976-1981 or in 1980-1989. In subsequent
TABLE 1 Characteristics of the $513 \mathrm{HBsAg}$ chronic carriers

\begin{tabular}{|c|c|}
\hline \multicolumn{2}{|l|}{ Variables } \\
\hline Age, years, $\mathrm{M} \pm \mathrm{SD}$ (range) & $57.8 \pm 13.7(16-88)$ \\
\hline \multicolumn{2}{|l|}{ Gender } \\
\hline Male/female & $330 / 183$ (Sex ratio 1.8) \\
\hline \multicolumn{2}{|l|}{ Referral pattern, $N^{\circ}(\%)$} \\
\hline In-patients & 59 (11.5) \\
\hline Out-patients & $454(88.5)$ \\
\hline \multicolumn{2}{|l|}{ Alcohol intake, $N^{\circ}(\%)$} \\
\hline Abstinent & $387(75.4)$ \\
\hline$\leq 4$ drinks/day & $42(8.2)$ \\
\hline$>4$ drinks/day & $84(16.4)$ \\
\hline \multicolumn{2}{|l|}{ Area of origin (birth), $N^{\circ}(\%)$} \\
\hline North & 65 (12.7) \\
\hline Center & $40(7.8)$ \\
\hline South/islands & $370(72.1)$ \\
\hline Abroad & $38(7.4)$ \\
\hline Years of awareness of HBsAg positivity & NA \\
\hline Antiviral therapy & NA \\
\hline \multicolumn{2}{|l|}{ Diagnostic category, $N^{\circ}(\%)$} \\
\hline Inactive carrier & $17(3.3)$ \\
\hline Chronic hepatitis & $326(63.4)$ \\
\hline Compensated cirrhosis & $118(23.0)$ \\
\hline Hepatocellular carcinoma & $24(4.7)$ \\
\hline Decompensated cirrhosis & $28(5.5)$ \\
\hline
\end{tabular}

NA, data not available.

studies from 2001 to 2014, the percentages of anti-HDV-positive subjects remained stable at around $11 \%$, while the prevalence of anti-HCV-positive cases decreased from $16.8 \%$ in 2001 to $6.5 \%$ in 2006-2007 and then increased to $10.7 \%$ in 2014 . The percentage of HBeAg-positive cases was $13.6 \%$ in the 2001 and $11.0 \%$ in the $2006 / 2007$ cases, but it had dropped to $6.2 \%$ by 2014 . The proportion of HBV inactive carriers progressively decreased over time from $46.9 \%$ to $3.3 \%(P<0.01)$, that of patients with chronic hepatitis increased from 32.1 to $63.4 \%(P<0.01)$, and that of patients with liver cirrhosis (with or without $\mathrm{HCC}$ ) remained stable at around $20 \%$ from the 1970 s to $2006-2007$, but it had significantly peaked at $33.2 \%$ by $2014(P<0.01)$ (Table 3$)$.

TABLE 2 Virological characteristics of all $513 \mathrm{HBsAg-positive}$ patients

\begin{tabular}{lll} 
Viral markers & N $^{\circ}$ positive/No tested & $\%$ \\
\hline HBeAg-positive & $32 / 513$ & 6.2 \\
\hline Anti-HDV & $61 / 513$ & 11.9 \\
\hline Anti-HCV-positive & $47 / 440^{\mathrm{a}}$ & 10.7 \\
\hline
\end{tabular}

an 73 subjects the test was not performed. 
TABLE 3 Characteristics of HBsAg chronic carriers in Italy before and after the vaccination campaign launched in 1991

\begin{tabular}{|c|c|c|c|c|c|}
\hline Years of observation & $1976-81$ Ref. $n^{\circ} 1$ & $1980-89$ Ref. $n^{\circ} 2$ & 2001 Ref. $n^{\circ} 3$ & 2006-07 Ref. $n^{\circ} 28$ & 2014 Ref. $n^{\circ} 10$ \\
\hline Number of patients & 700 & 1710 & 1336 & 1386 & 513 \\
\hline Age, years, $M \pm S D$ & $27 \pm 16$ & $30.8 \pm 17.2$ & $49.1 \pm 15.9$ & $49.7 \pm 12.8$ & $57.8 \pm 13.7$ \\
\hline HBeAg-positive, $N^{\circ}(\%)$ & NA & NA & $154 / 1133(13.6)$ & $148 / 1348(11.0)$ & $32(6.2)$ \\
\hline Anti-HDV-positive, $N^{\circ}(\%)$ & NA & NA & $61 / 723(9.7)$ & 95/1179 (8.2) & $61(11.9)$ \\
\hline \multicolumn{6}{|l|}{ Diagnostic category, $N^{\circ}(\%)$} \\
\hline Inactive carrier & $328(46.9)$ & 655 (38.3) & $267(20.0)$ & $184(13.3)$ & $17(3.3)$ \\
\hline Chronic hepatitis & $225(32.1)$ & 669 (39.1) & $768(57.5)$ & $871(62.8)$ & $326(63.4)$ \\
\hline Cirrhosis & $153(21.9)$ & $386(22.6)$ & $248(18.6)$ & $291(21.0)$ & $146(28.5)$ \\
\hline
\end{tabular}

NA, data not available.

Table 4 shows the distribution of the $\mathrm{HBsAg}$-positive patients observed in the 2001, 2006-2007, and 2014 studies by age class (0-33, 34-59, and $>60$ ) and diagnosis (Table 4). The percentage of inactive carriers aged $0-33$ years decreased from $21.7 \%$ in 2001 to $5.9 \%$ in 2014 and a similar downtrend was observed in patients with chronic hepatitis (from 17.5 to $5.2 \%$ ), whereas the rate of cases with cirrhosis ranged from $5 \%$ to $8 \%$ in the different studies and no cirrhotic patient had superimposed HCC. Conversely, these percentages increased from 2001 to 2014 in the over-60 age sub-group (Table 4). To this regard, it should be stated that the universal $\mathrm{HBV}$ vaccination covered Italian citizens aged 0-10 and 12-21 in 2001, 0-26 in 2006-2007 and 0-33 in 2014, and that in the earliest years of application a $65.1 \%$ vaccine coverage had been registered in adolescents in some southern districts.

\section{4 | DISCUSSION}

One of the major aims of the HBV vaccination campaign was the reduction in the burden of $\mathrm{HBsAg}$-positive CLD, including $\mathrm{HCC}$. Population surveys performed in Taiwan on children born before and after the introduction of universal HBV vaccination showed a drastic decrease in the HBV carrier rate and infection rate among subjects born since the program began. ${ }^{16}$ Moreover, studies on the secular trend of liver disease documented a $68 \%$ decline in mortality from fulminant hepatitis in infants and a 75\% decrease in the incidence of HCC in children 6-9 years old after the introduction of a national vaccination program. ${ }^{17}$ Similarly, substantial reductions in newly acquired HBV infections, $\mathrm{HBsAg}$ carrier rate and HBV-related mortality have been reported in countries where universal vaccination has been implemented, such as Alaska ${ }^{18}$ and Gambia. ${ }^{19}$

In Italy, the data from the national surveillance system for viral hepatitis (SEIEVA) indicate a marked decrease in the incidence of acute $B$ hepatitis in the age-groups covered by vaccination (Fig. 1). This is in good agreement with the results of a sero-epidemiological survey performed in 2010 in a Southern Italian town, ${ }^{20}$ where no subject below 30 years was anti-HBc-positive, thus confirming the effectiveness of the vaccination program. Indeed, vaccination coverage in Italy ranged from $95 \%$ to $98 \%$ in different years of application.

Comparing the age distribution of chronic HBsAg carriers from the three different surveys, we evaluated the impact of vaccination on the burden of HBsAg-positive CLD over a 23-year period. The data showed a progressive, significant downtrend in the prevalence of chronic HBsAg carriers in the age group covered by vaccination.

The increasing coverage of HBV vaccination has minimized the proportion of young subjects with HBV-related CLD, generating over time an increase in the mean age of chronic HBsAg carriers. In the prevaccination era the mean age of patients with HBV-related CLD was 27.0 years in those enrolled in 1976-1981 and 30.8 years in those investigated in 1980-1989. The mean age of these patients was 49.1 years in 2001, when HBV vaccination covered all Italian citizens aged 0-10 and 12-21, 49.7 in 2006-2007, when HBV vaccination covered the age group $0-26$, and 58.9 years in 2014 , when virtually all the Italian citizens aged 0-33 were covered by HBV vaccination. However, $5.9 \%$ of $\mathrm{HBsAg}$ inactive carriers, $5.2 \%$ of patients with chronic hepatitis, and $4.1 \%$ of those with cirrhosis who eluded vaccination is still present in the age group 0-33 years. The low vaccine coverage (65.1\%) among adolescents in southern areas of the country during the earliest years of the campaign, documented in a national survey performed in 136 Italian health districts, ${ }^{21}$ may account for this.

The remarkable shift toward older age groups has generated important virological and clinical changes. The rate of $\mathrm{HBeAg}$ positivity has progressively declined in Italy from $58.4 \%$ detected in 356 patients investigated in $1975-1985^{22}$ to $20.1 \%$ found in a multicenter study performed in $1990,{ }^{23}$ to $13.6 \%$ reported in $2001,{ }^{24} 11 \%$ in $2006-2007,{ }^{25}$ and $6.2 \%$ in the present investigation in 2014. Before the vaccination was introduced, HBV/HDV chronic coinfection was $23.4 \%$ in $1987^{26}$ and $14.5 \%$ in $1992 .{ }^{27}$ Subsequently, the prevalences ranged from $8.3 \%$ in $1997^{28}$ to $11.9 \%$ in $2014,{ }^{29}$ mostly as a consequence of the impact of vaccination. Of note, all the anti-HDV-positive cases were over 50 years of age and half of them had cirrhosis; this pattern strongly supports the concept that HDV circulation has drastically decreased among Italian 


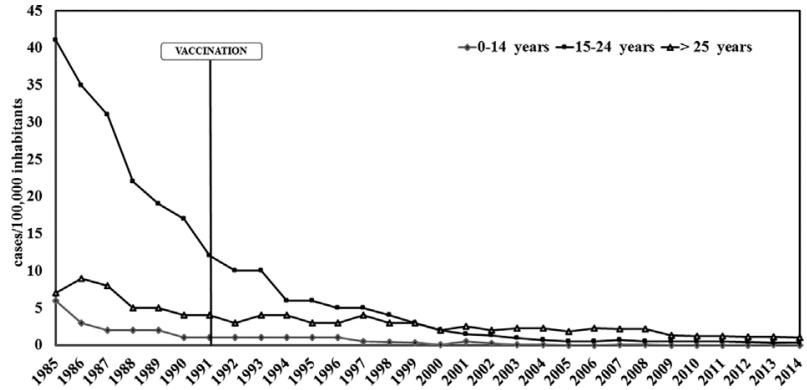

FIGURE 1 Age-specific incidence (n. cases $\times 100000$ inhabitants) of acute hepatitis B in Italy, 1985-2014. (Source of data: SEIEVA)

patients, and the current prevalence of anti-HDV positivity reflects a "survival effect"; HDV infection in Italy is an aging disease with a long-term course.

Surprisingly, the prevalence of $\mathrm{HBV} / \mathrm{HCV}$ coinfection increased from $6.5 \%$ in $2006-2007$ to the current $10.7 \%$, a trend most probably due to a referral bias involving increased HCV testing at referral liver units due to the recent availability of free of charge second generation DAAs for HCV eradication.

In conclusion, this study provides evidence of the effectiveness of universal HBV vaccination in preventing chronic liver disease among the youngest generations 23 years after its launch in Italy.

\section{CONFLICTS OF INTEREST}

All the authors of this manuscript declare they have no conflicts of interest in connection with this paper.

\section{ORCID}

Evangelista Sagnelli (iD http://orcid.org/0000-0003-2817-8436

\section{REFERENCES}

1. Giusti G, Ruggiero G, Galanti B, Piccinino F, Sagnelli E, Gallo C. Chronic active hepatitis in Italy: a multicentric study on clinical and laboratory data of 1154 cases. Hepato-Gastroenterology. 1983;30:126-130.

2. Giusti G, Sagnelli E, Gallo C, Piccinino F, Galanti B, Gaeta GB. The etiology of chronic hepatitis in Italy: a multicentric study. Hepatogastroenterol. 1994;41:397-400.

3. Sagnelli E, Stroffolini T, Mele A, et al. The importance of HCV on the burden of chronic liver disease in Italy: a multicenter prevalence study on 9,997 cases. J Med Virol. 2005;75:522-527.

4. Sagnelli E, Stroffolini T, Sagnelli C, et al. Almasio PL; EPACRON study Group. Epidemiological and clinical scenario of chronic liver diseases in Italy: data from a multicenter nationwide survey. Dig Liver Dis. 2016;30431-30435.

5. Gaiani S, Gramantieri L, Venturoli N, et al. What is the criterion for differentiating chronic hepatitis from compensated cirrhosis? A prospective study comparing ultrasonography and percutaneous liver biopsy. J Hepatol. 1997;27:979-985. 
6. Bruix J, Sherman M, Llovet JM, et al. Clinical management of hepatocellular carcinoma. Conclusions of the Barcelona-2000 EASL conference. European Association for the Study of the Liver.J Hepatol 2001;35:421-430.

7. Becker U, Deis A, Sorensen $\mathrm{TI}$, et al. Prediction of risk of liver disease by alcohol intake, sex and age. A prospective population study. Hepatology 1996;23:1025-1029.

8. Mc Cullough AJ, O' Connor JEB. Alcoholic liver disease: proposed recommendation for the American College of Gastroenterology. Am J Gastroenterol. 1998;93:2022-2026.

9. Ishak K, Baptista A, Bianchi L, et al. Histological grading and staging of chronic hepatitis. J Hepatol. 1995;22:696-699.

10. Bedossa P, Poynard T. An algorithm for the grading of activity in chronic hepatitis C. The METAVIR Cooperative Study Group. Hepatology 1996;24:289-293.

11. Gamal S, Khaled Z. 2011. In: Dr. Takahashi Hirokazu, editor. Ishak versus METAVIR: Terminology, Convertibility and Correlation with Laboratory Changes in Chronic Hepatitis C, Liver Biopsy. ISBN: 978953-307-644-7. Available from: http://www.intechopen.com/books/ liver-biopsy/ishak-versus-metavir-terminology-convertibility-andcorrelation-with-laboratory-changes-in-chronic-h

12. Fraquelli M, Rigamonti $C$, Casazza $G$, et al. Etiology-related determinants of liver stiffness values in chronic viral hepatitis B or C. J Hepatol. 2011;54:621-628.

13. Chon YE, Choi EH, Song KJ, et al. Performance of transient elastography for the staging of liver fibrosis in patients with chronic hepatitis B: A meta-analysis. PLoS ONE 2012;7:e44930.

14. Wong VW, Vergniol J, Wong GL, et al. Liver stiffness measurement using $\mathrm{XL}$ probe in patients with nonalcoholic fatty liver disease. Am J Gastroenterol. 2012;107:1862-1871.

15. Coppola N, Sagnelli C, Pisaturo M, et al. Clinical and virological characteristics associated with severe acute hepatitis B. Clin Microbiol Infect. 2014;20:0991-0997.

16. Ni YH, Chang MH, Wu JF, Hsu HY, Chen HL, Chen DS. Minimization of hepatitis $\mathrm{B}$ infection by a 25 -year universal vaccination program. J Hepatol. 2012;57:730-735.

17. Chien YC, Jan CF, Kuo HS, Chen CJ. Nationwide hepatitis B vaccination program in Taiwan: effectiveness in the 20 years after it was launched. Epidemiol Rev. 2006;28:126-135.

18. Harpaz R, McMahon BJ, Margolis HS, et al. Elimination of new chronic hepatitis B virus infections: results of the Alaska immunization program. J Infect Dis. 2000;181:413-418.
19. Whittle H, Jaffar S, Wansbrough M, Mendy M, Dumpis U. Observational study of vaccine efficacy 14 years after trial of hepatitis B vaccination in Gambian children. BMJ. 2002;325:569.

20. Stroffolini T. The impact of a vaccination campaign against hepatitis $B$ on the further decrease of hepatitis $B$ virus infection in a southern Italian town over 14 years. Eur J Intern Med. 2012;23:190-192.

21. Stroffolini T, Almasio PL, Sagnelli E, Mele A. Gaeta GB, Mele A. Vaccination campaign against hepatitis $B$ for 12-year-old subjects in Italy. Vaccine. 1997;15:583-585.

22. Giusti G, Galanti B, Gaeta GB, Sagnelli E, Piccinino F, Ruggiero G. Clinical presentation and natural history of chronic persistent hepatitis. A multicentre retrospective study on 1197 cases. Ital J Gastroenterol. 1991;23:111-118.

23. Rapicetta M, di Nardo V, Rozera C, et al. HBV-DNA, HBeAg/anti-HBe serological status in hepatitis $B$ chronic individuals from central Italy. Epidemiol Infect. 1990;104:511-517.

24. Sagnelli E, Stroffolini T, Mele A, Imparato M, Almasio PL. Chronic hepatitis B in Italy: new features in an old disease - Approaching the universal prevalence of Hepatitis $B$ e antigen-negative cases and the eradication of Hepatitis D infection. Clin Infect Dis. 2008;46:110-113.

25. Stroffolini T, Almasio PL, Sagnelli E, Mele A. Gaeta GB; italian hospitals' collaborating group. evolving clinical landscape of chronic hepatitis B: a multicenter italian study. J Med Virol. 2009;81:1999-2006.

26. Sagnelli E, Stroffolini T, Ascione A, et al. The epidemiology of hepatitis delta infection in Italy Promoting Group. J Hepatol. 1992;15:211-215.

27. Sagnelli E, Stroffolini T, Ascione A, et al. Decrease in HDV endemicity in Italy. J Hepatol. 1997;26:20-24.

28. Gaeta GB, Stroffolini T, Chiaramonte M, et al. Chronic hepatitis D: a vanishing disease? an italian multicenter study. Hepatology. 2000;32:824-827.

29. Stroffolini T, Sagnelli E, Sagnelli C, et al. Hepatitis delta infection in Italian patients: towards the end of the story? Infection. 2016;107:1862-1871.

How to cite this article: Sagnelli E, Stroffolini T, Sagnelli C, et al. Influence of universal HBV vaccination on chronic HBV infection in Italy: Results of a cross-sectional multicenter study. J Med Virol. 2017;89:2138-2143.

https://doi.org/10.1002/jmv.24873 